TOKYO J. MATH.

Vol. 19, No. 1, 1996

\title{
Complete Intersections Which Are Abelian Extensions of a Factorial Domain
}

\author{
Yuji KAMOI \\ Tokyo Metropolitan University \\ (Communicated by T. Ishikawa)
}

\section{Introduction.}

Let $A$ be a Noetherian local factorial domain having a field of fractions $K$ and $L$ be an Abelian extension of $K$ with $G=G a l(L / K)$. An Abelian extension $R$ of $A$ is an integral closure of $A$ in $L$.

Assume that $\operatorname{ch}(A)$ does not divide $n=|G|$ and $A$ has a primitive $n$-th root of unity. Roberts [12] showed that $R$ is Cohen-Macaulay, if $\boldsymbol{A}$ is a Cohen-Macaulay factorial domain. Also Itoh [8] studied a condition for a cyclic extension of a formal power series ring to be Gorenstein. Furthermore, Griffith [7] showed that if $A$ is regular and if $R$ is factorial, then $R$ is a complete intersection.

Our purpose of this article is to give a condition for $R$ to be a complete intersection. We shall show in section 4 that an Abelian extension of a local factorial domain $\boldsymbol{A}$ which is a complete intersection, is completely determined by a datum of $A$. A datum is a pair $(\Gamma, w)$ of a finite subset $\Gamma$ of $\operatorname{Div}(A) \otimes_{\mathrm{z}} \mathbf{Q}\left(=\mathrm{P}(A) \otimes_{\mathrm{z}} \mathbf{Q}\right.$ since $A$ is factorial $)$ and a map $w: \Gamma \rightarrow \mathbf{N}_{+}$satisfying the following condition.

(1) For $D, E \in \Gamma(D \neq E)$, one of the following cases occurs;

(a) $\operatorname{Supp}(D) \subsetneq \operatorname{Supp}(E)$, (b) $\operatorname{Supp}(D) \supsetneq \operatorname{Supp}(E)$, (c) $\operatorname{Supp}(D) \cap \operatorname{Supp}(E)=\varnothing$.

(2) For $E \in \Gamma$, there is a relation $w(E) E=\sum_{i=1}^{k} E_{i}+\sum_{j=1}^{l} \mathfrak{p}_{j}$ where $\left\{E_{1}, \cdots, E_{k}\right\}=$ $\{D \in \Gamma \mid D<E\}$ and $\left\{\mathfrak{p}_{1}, \cdots, \mathfrak{p}_{l}\right\}=\operatorname{Supp}(E) \backslash \bigcup_{i=1}^{k} \operatorname{Supp}\left(E_{i}\right)$.

Here we denote by $\operatorname{Supp}(D)\left(D \in \operatorname{Div}(A)_{\mathbf{Q}}\right)$ the set of prime divisors of $A$ which appear in $D$ with non-zero coefficients. We write $D \prec E$, if $\operatorname{Supp}(D) \subsetneq \operatorname{Supp}(E)$ and if there is no element $D^{\prime} \in \Gamma$ such that $\operatorname{Supp}(D) \subsetneq \operatorname{Supp}\left(D^{\prime}\right) \subsetneq \operatorname{Supp}(E)$.

Then we state our main result as follows.

THEOREM. Let $A$ be as above and $R$ be a local ring such that $R \supset A$. Then the following conditions are equivalent.

(1) $R$ is an Abelian extension of $A$ which is a complete intersection.

Received August 12, 1994 
(2) There exists a datum $(\Gamma, w)$ such that

$$
R \cong A\left[Y_{E} \mid E \in \Gamma\right] /\left(Y_{E}^{w(E)}-a_{E} \prod_{D<E} Y_{D} \mid E \in \Gamma\right)
$$

where $A\left[Y_{E} \mid E \in \Gamma\right]$ is a polynomial ring and $a_{E}$ is an element of $A$ satisfying $\operatorname{div}\left(a_{E}\right)=$ $w(E) E-\sum_{D \prec E} D$.

A datum was first defined by Watanabe [15] for normal simplicial semigroup rings (cf. Definition 1.1 of [15]). He used it to determine normal simplicial semigroup rings which are complete intersections. In his case, an affine semigroup ring can be regarded as a $\mathbf{Z}^{\mathrm{n}}$-graded ring in natural way. In our case, an Abelian extension $R$ is graded by its Galois group. (See (4.1).) By this reason, the concept of rings graded by an Abelian group is essential for us. In particular, we state a group graded version of the divisor group theory (in section 1).

\section{Preliminaries.}

Let us recall some concepts of graded rings and graded modules from [6], [11] and [9].

Let $G$ be an Abelian group. We say that a ring $R$ is a $G$-graded ring, if there exists a family $\left\{R_{g}\right\}_{g \in G}$ of additive subgroups of $R$ such that $R=\bigoplus_{g \in G} R_{g}$ and $R_{g} R_{h} \subset R_{g+h}$ for every $g, h \in G$. Similarly, a $G$-graded $R$-module is an $R$-module $M$ for which there is given a family $\left\{M_{g}\right\}_{g \in G}$ of additive subgroups of $M$ such that $M=\bigoplus_{g \in G} M_{g}$ and $R_{g} M_{h} \subset M_{g+h}$ for every $g, h \in G$.

A homomorphism $f: M \rightarrow N$ of $G$-graded $R$-modules in an $R$-linear map such that $f\left(M_{g}\right) \subset N_{g}$ for all $g \in G$.

Let $R$ be a $G$-graded ring and $M$ a $G$-graded $R$-module. For $g \in G$, we define a $G$-graded $R$-module $M(g)$ by $M=M(g)$ as the underlying $R$-module and graded by $[M(g)]_{h}=M_{g+h}$ for all $h \in G$. We say that $M$ is free, if it is isomorphic to a direct sum of $G$-graded $R$-modules of the form $R(g)(g \in G)$.

We denote by $\underline{\operatorname{Hom}}_{R}(M, N)_{g}$ the Abelian group of all the $G$-graded homomorphisms from $M$ to $N(g)$. We put $\underline{\operatorname{Hom}}_{R}(M, N)=\bigoplus_{g \in G} \underline{\operatorname{Hom}}_{R}(M, N)_{g}$ and consider it as a $G$-graded $R$-module.

The elements of $\bigcup_{g \in G} M_{g}$ are called homogeneous elements of $M$. Every non-zero element $x \in M_{g}$ is said to be homogeneous of degree $g$, and we denote $\operatorname{deg}(x)=g$. For a subset $N \subset M$, we set $h(N)=\bigcup_{g \in G}\left(N \cap M_{g}\right)$. Any element $x \in M$ has a unique expression as a sum of homogeneous elements, $x=\sum_{g \in G} x_{g}$ where $x_{g} \in M_{g}$ and $x_{g}=0$ for almost all $g \in G$. With this notation, we call nonzero $x_{g}$ the homogeneous component (of degree $g$ ) of $x$.

Let $H$ be a subgroup of $G$ and $g \in G$. We define $R^{(H)}=\bigoplus_{h \in H} R_{h}$ and $M^{(g, H)}=$ $\bigoplus_{h \in H} M_{g+h^{*}}$. Then $R^{(H)}$ is a subring of $R$ and $M^{(g, H)}$ is an $R^{(H)}$-submodule of $M$. We 
define a $G$-grading on $M^{(g, H)}$ as

$$
\left[M^{(g, H)}\right]_{g^{\prime}}=\left\{\begin{array}{lll}
M_{g^{\prime}}, & \text { if } & g-g^{\prime} \in H \\
(0), & \text { if } & g-g^{\prime} \notin H
\end{array}\right.
$$

for all $g^{\prime} \in G$. If $g-g^{\prime} \in H$, then we have $M^{(g, H)}=M^{\left(g^{\prime}, H\right)}$ as a $G$-graded $R^{(H)}$-module. Hence $M$ has the following decomposition as a $G$-graded $R^{(H)}$-module

$$
M=\bigoplus_{i \in \Gamma} M^{\left(g_{i}, H\right)}
$$

where $\left\{g_{i}\right\}_{i \in \Gamma}$ is a system of representatives of $G \bmod H$. Also, we have $R^{\left(g_{i}, H\right)} M^{\left(g_{j}, H\right)} \subset$ $M^{\left(g_{i}+g_{j}, H\right)}$ for all $i, j \in \Gamma$. Hence a $G$-graded ring $R$ (resp. $G$-graded $R$-module $M$ ) can be regarded as a $G / H$-graded ring (resp. $G / H$-graded $R$-module).

We say that $R$ is a $G$-domain (resp. $G$-simple), if every nonzero $G$-homogeneous element of $R$ is a nonzero divisor of $R$ (resp. a unit of $R$ ).

A $G$-graded ideal $\mathfrak{P}$ of $R$ is said to be a $G$-prime ideal (resp. a $G$-maximal ideal), if the $G$-graded ring $R / \mathfrak{P}$ is a $G$-domain (resp. $G$-simple). Note that a $G$-prime ideal is not necessarily a prime ideal, if $G$ has a torsion. We denote by $V_{G}(R)$ the set of all $G$-prime ideals of $R$. For $\mathfrak{B} \in V_{G}(R)$, we denote by $M_{(\mathfrak{B})}$ the module of fractions of $M$ with respect to the multiplicatively closed subset $h(R \backslash \mathfrak{P})$ and call it the homogeneous localization of $M$ at $\mathfrak{P}$. We set $V_{G}(M)=\left\{\mathfrak{P} \in V_{G}(R) \mid M_{(\mathfrak{P})} \neq(0)\right\}$. For an ideal $P$ of $R$, we denote by $P^{*}$ the maximal graded ideal of $R$ contained in $P$ (or the graded ideal generated by $h(P)$ ). If $P$ is a prime ideal of $R$, then $P^{*}$ is a $G$-prime ideal of $R$. Furthermore, for a $G$-graded $R$-module $M$ and $P \in \operatorname{Spec}(R), P \in \operatorname{Supp}_{R}(M)$ if and only if $P^{*} \in V_{G}(M)$. We denote by $\underline{\operatorname{dim}}(M)$ the largest length of a chain of $G$-prime ideals in $V_{G}(M)$ (cf. section 2 of [9]).

REMARK 1.1. Let $H$ be a subgroup of $G$ such that $G / H$ is torsion. If $\mathfrak{B} \in V_{G}(R)$, then $\mathfrak{P}^{(H)} \in V_{H}\left(R^{(H)}\right)$. Furthermore, if $\mathfrak{p} \in V_{H}\left(R^{(H)}\right)$, then a $G$-graded ideal $(\sqrt{\mathfrak{p} R})^{*}$ is $G$-prime. This gives a bijective correspondence between $V_{H}\left(R^{(H)}\right)$ and $V_{G}(R) . M_{(\mathfrak{P})} \cong$ $M \otimes_{R^{(H)}}\left(R^{(H)}\right)_{(\mathfrak{P}(H))}$ holds for a $G$-graded $R$-module $M$ and $\mathfrak{P} \in V_{G}(R)$.

DEFINITION 1.2. $R$ is said to be a $G$-Noetherian graded ring, if every strictly ascending chain of $G$-graded ideals of $R$ has finite length.

Definition 1.3. We say that $R$ is a $G$-local graded ring, if it has a unique $G$-maximal ideal. Often we use the notation $(R, \mathfrak{M})$ to say that $R$ is a $G$-local ring with the unique $G$-maximal ideal $\mathfrak{M}$.

Next, we state a $G$-graded version of the theory of divisors. All propositions shall be proved in the same way as in the non graded case or $\mathbf{Z}^{n}$-graded case (cf. Anderson [1]). Therefore, we omit proofs.

Let $R$ be a $G$-domain and $K$ be the homogeneous localization of $R$ at (0). 
DEFINITION 1.4. A $G$-domain $R$ is called $G$-normal, if every element of $h(K)$, which is integral over $R$, is in $R$.

DEFINITION 1.5. $R$ is said to be completely $G$-normal, if it satisfies the following condition; if $x \in h(K)$ and $R[x]$ is contained in a finitely generated $G$-graded $R$-submodule of $K$, then $x \in R$.

A $G$-graded $R$-submodule $0 \neq I$ of $K$ is said to be a $G$-fractional ideal of $R$, if there exists $a \in R$ such that $a I \subset R$. We denote by $\underline{\mathrm{I}}(R)$ the set of all $G$-fractional ideals of $R$ and by $\underline{\mathrm{P}}(R)$ the set of all homogeneous principal idelas. We set $\underline{\operatorname{div}}_{R}(I)=$ $\bigcap_{0 \neq x \in h(K), I \subset R_{x}} R_{x}$ for $I \in \underline{\mathrm{I}}(R)$.

DEFINITION 1.6. A $G$-fractional ideal $I$ is said to be a $G$-divisorial ideal of $R$, if $I=\underline{\operatorname{div}}_{R}(I)$. We denote by $\underline{\operatorname{Div}}(R)$ the set of all $G$-divisorial ideals of $R$.

REMARK 1.7. Let $I, J \in \underline{\mathrm{I}}(R)$. Then the following hold.

(0) $\operatorname{Hom}_{R}(I, J) \cong[J: I]$.

(1) $[I: J]_{K}=\bigcap_{x \in h(J)} x^{-1} I$.

(2) If $I \in \underline{\operatorname{Div}}(R)$, then so is $[I: J]_{K}$.

(3) $\operatorname{div}_{R}(I)=\left[R:[R: I]_{K}\right]_{K}$.

(4) $\underline{\operatorname{div}}_{R}(I) \subset \underline{\operatorname{div}}_{R}(J)$ if and only if $[R: I]_{K} \supset[R: J]_{K}$.

DEFINITION 1.8. We define a commutative monoid structure on $\underline{\operatorname{Div}}(R)$ by $\underline{\operatorname{div}}_{R}(I)+\underline{\operatorname{div}}_{R}(J):=\underline{\operatorname{div}}_{R}(I J)$ for $I, J \in \underline{\mathrm{I}}(R)$. Also, we $\operatorname{denote} \underline{\operatorname{div}}_{R}(I) \sim \underline{\operatorname{div}}_{R}(J)$, if $\underline{\operatorname{div}}_{R}(I)=\underline{\operatorname{div}}_{R}(J)+\underline{\operatorname{div}}_{R}(a)$ for some $0 \neq a \in h(K)$. Here we denote $\underline{\operatorname{div}}_{R}(a)=\underline{\operatorname{div}}_{R}(a R)$ for $0 \neq a \in h(K)$. We put $\underline{C l}(R)=\underline{\operatorname{Div}}(R) / \sim$.

The following proposition is proved in the same way as in the non-graded case (cf. Chap. VII, §1.2, Theorem 1 of [3]).

Proposition 1.9. $R$ is completely G-normal if and only if $\underline{\operatorname{Div}}(R)$ is an Abelian group.

Definition 1.10. Let $\Gamma$ be an ordered Abelian group and $v: h(K \backslash\{0\}) \rightarrow \Gamma$ be a map. We call $v$ a $G$-valuation on $K$, if it satisfies the following two conditions; for $x, y \in h(K \backslash\{0\})$,

(1) $v(x y)=v(x)+v(y)$

(2) $v(x+y) \geq \min (v(x), v(y))$, if $\operatorname{deg}(x)=\operatorname{deg}(y)$ and $x+y \neq 0$.

Let $v$ be a $G$-valuation on $K$. Then $\left.v\right|_{K_{0}^{\prime}}$ is a valuation on $K_{0}^{\prime}:=K_{0} \backslash\{0\}$. We denote by $R_{v 0}$ the valuation ring of $\left.v\right|_{K_{0}^{\prime}}$. We set $R_{v}=R_{v 0}[x \in h(K \backslash\{0\}) \mid v(x) \geq 0]$ and call it the $G$-valuation ring of $v$.

A $G$-valuation $v: h(K \backslash\{0\}) \rightarrow \Gamma$ is said to be equivalent to a $G$-valuation $v^{\prime}$ : $h(K \backslash\{0\}) \rightarrow \Gamma^{\prime}$, if there exists an isomorphism $\phi: \operatorname{Im}(v) \rightarrow \operatorname{Im}\left(v^{\prime}\right)$ of ordered Abelian groups such that $v^{\prime}=\phi \circ v$. 
DEFINITION 1.11. A $G$-valuation $v$ on $K$ is said to be discrete, if $\operatorname{Im}(v) \cong \mathbf{Z}$.

We say that $R$ is a $G$-discrete valuation ring $(G-D V R)$, if there exists a discrete $G$-valuation $v$ on $K$ such that $R=R_{v}$.

As in the non-graded case, the following hold.

Proposition 1.12. Let $(R, \mathfrak{M})$ be a G-Noetherian G-local G-domain of $\underline{\operatorname{dim}}(R)=1$. Then the following are equivalent.

(1) $R$ is $G-D V R$.

(2) $R$ is G-normal.

(3) $\mathfrak{M}$ is a principal ideal.

(4) Every proper G-homogeneous ideal is a power of $\mathfrak{M}$.

EXAMPLE 1.13. Let $(R, \mathfrak{M})$ be a $G$-DVR and $x \in h(R)$ such that $\mathfrak{M}=x R$. We put $H=\left\{g \in G \mid R_{g} \neq \mathfrak{M}_{g}\right\} \quad\left(=\left\{g \in G \mid R_{g}\right.\right.$ contains a unit of $\left.\left.R\right\}\right)$. (Note that $H$ is a subgroup of $G$.) Then $\mathfrak{M}^{(H)}=\mathfrak{M}_{0} R^{(H)}$ (cf. (1.4) of [9]).

- If $\mathfrak{M}_{0}=(0)$ (i.e. $\mathfrak{M}^{(H)}=(0)$ ), then $R^{(H)}$ is $H$-simple and $x^{n} \notin R^{(H)}$ for every $n>0$. Hence $n \operatorname{deg}(x) \notin H$ for every $n>0$ (or $H \oplus \mathbf{Z} \operatorname{deg}(x) \subset G$ ) and $R=R^{(H)}[x]$ the polynomial ring over $R^{(H)}$.

- If $\mathfrak{M}_{0} \neq 0$, then $\left(R_{0}, \mathfrak{M}_{0}\right)$ is a DVR. We take $x_{0} \in \mathfrak{M}_{0}$ such that $\mathfrak{M}_{0}=x_{0} R_{0}$. Then there exists a positive integer $e>0$ such that $x_{0}=u x^{e}$ for some homogeneous unit $u \in R^{(H)}$. Hence $R=\bigoplus_{i=0}^{e-1} R^{(H)} x^{i}$

EXAMPLE 1.14. Let $K=\bigoplus_{n \in \mathbf{Z}} K_{n}$ be a simple Z-graded ring. We assume that $K \neq K_{0}$. Then there exists $t \in h(K \backslash\{0\})$ such that $t$ is algebraically independent over $K_{0}$ and $K=K_{0}\left[t, t^{-1}\right]$. We define $\mathbf{Z}$-valuations as follows;

(i) $v_{t}: h(K \backslash\{0\}) \rightarrow \mathbf{Z}$ by $v_{t}\left(a t^{n}\right)=n\left(a \in K_{0}\right)$,

(ii) $v_{t^{-1}}: h(K \backslash\{0\}) \rightarrow \mathbf{Z}$ by $v_{t}\left(a t^{n}\right)=-n\left(a \in K_{0}\right)$,

(iii) $v_{0}^{d, e}: h(K \backslash\{0\}) \rightarrow \mathbf{Z}(1 / e)(\subset \mathbf{Q})$ by $v_{0}^{d, e}\left(a t^{n}\right)=v_{0}(a)+n(d / e)\left(a \in K_{0}\right)$ where $v_{0}$ is a discrete valuation of $K_{0}$ and $d, e \in \mathbf{Z}$ such that $e>0$ and either $d=0$ or $(e, d)=1$.

Let $v$ be a discrete $Z$-valuation of $K$ and $(R, x R)$ be a $Z$-valuation ring of $v$. We put $m \geq 0$ such that $\mathbf{Z} m=\left\{n \in \mathbf{Z} \mid R_{n}\right.$ contains a unit of $\left.R\right\}$. Then, by (1.13), either (1) $R=R^{(m)}[x]$ or (2) $R=\bigoplus_{i=0}^{e-1} R^{(m)} x^{i}$.

Case (1) Since $\mathbf{Z} m \oplus \mathbf{Z} \operatorname{deg}(x) \subset \mathbf{Z}, m=0$ and $R=R_{0}[x]$. On the other hand, $K$ is a homogeneous localization of $R$ at (0) (i.e. $K=R_{0}\left[x, x^{-1}\right]$ ). Hence $R_{0}=K_{0}$ and either $x=t$ or $x=t^{-1}$. Namely, either $v=v_{t}$ or $v=v_{t-1}$.

Case (2) We put $v(t)=d$ and $v_{0}=\left.(1 / e) v\right|_{K_{0}}$. Then $v_{0}$ is a normalized discrete valuation of $R_{0}$. Furthermore, for $a \in K_{0} \backslash\{0\}$ and $n \in \mathbf{Z}, v\left(a t^{n}\right)=e\left(v_{0}(a)+n(d / e)=\right.$ $e v_{0}^{d, e}\left(a t^{n}\right)$. Hence $v$ is equivalent to $v_{0}^{d, e}$ and $R=R_{v_{0}^{d, e}}=\bigoplus_{n \in \mathbf{Z}} R_{0} x_{0}^{[-n d / e]} t^{n}$ where $x_{0}$ is the primitive element of $R_{0}$ and [a] is the largest integer not larger than $a$ for $a \in \mathbf{Q}$.

We set $V_{G}^{1}(R)=\left\{\mathfrak{P} \in V_{G}(R) \mid \underline{\operatorname{dim}}\left(R_{(\mathfrak{P})}=1\right\}\right.$. 
Definition 1.15. A $G$-domain $R$ is said to be $G$-Krull, if it satisfies the following conditions:

(1) $R_{(\mathfrak{P})}$ is $G$-DVR for every $\mathfrak{P} \in V_{G}^{1}(R)$,

(2) $R=\bigcap_{\mathfrak{B} \in V_{G}^{1}(R)} R_{(\mathfrak{P})}$,

(3) every nonzero element of $h(R)$ is contained in only a finite members of $V_{G}^{1}(R)$.

Proposition 1.16 (cf. Chap. VII, §1 of [3]). (1) A G-domain $R$ is $G$-Krull, if $R$ is $G$-Noetherian and $G$-normal. Conversely, a $G$-Krull $R$ is completely $G$-normal.

(2) A G-graded Krull domain is G-Krull.

(3) Let $R$ be a $G$-Krull $G$-domain and $S$ be a multiplicatively closed subset of $h(R)$. Then a G-graded ring $S^{-1} R$ is $G$-Krull and $S^{-1} R=\bigcap_{\mathfrak{B} \in A} R_{(\mathfrak{P})}$ where $\Lambda=\{\mathfrak{P} \in$ $\left.V_{G}^{1}(R) \mid \mathfrak{P} \cap S=\varnothing\right\}$.

(4) Let $R$ be a $G$-Krull $G$-domain and $K$ be the homogeneous localization of $R$ at (0). If $K^{\prime}$ is a simple graded subring of $K$, then $R \cap K^{\prime}$ is a G-Krull G-domain. In particular, $R^{(H)}$ is an $H$-Krull $H$-domain for a subgroup $H$ of $G$.

Throughout this section, we assume that $R$ is $G$-Krull.

Proposition 1.17 (cf. Chap. VII, $\$ 1$ of [3]). (1) For $I \in \underline{I}(R), \underline{\operatorname{div}}_{R}(I)=$ $\bigcap_{\mathfrak{P} \in V_{G}^{1}(R)} I R_{(\mathfrak{P})}$.

(2) For $I, J \in \underline{\operatorname{Div}}(R), I=J$ if and only if $I_{(\mathfrak{P})}=J_{(\mathfrak{P})}$ for every $\mathfrak{B} \in V_{G}^{1}(R)$.

COROllary 1.18. Let $H$ be a subgroup of $G$ such that $G / H$ is torsion and $\left\{g_{i}\right\}_{i \in \Gamma}$ be a system of representatives of $G \bmod H$. Then a $G$-fractional ideal $I$ is $G$-divisorial if and only if $I^{\left(g_{i}, H\right)}$ is $H$-divisorial for any $i \in \Gamma$.

Let $\mathfrak{P} \in V_{G}^{1}(R)$. Then, by $(1.12), \mathfrak{P} R_{(\mathfrak{P})}$ defines a discrete $G$-valuation on $K$. We denote it by $v_{\mathfrak{P}}$.

We define a map $\mu: \underline{\operatorname{Div}}(R) \rightarrow \bigoplus_{\mathfrak{P} \in V_{G}^{1}(R)} \mathbf{Z P}$ by $\mu\left(\underline{\operatorname{div}}_{R}(I)\right)=\sum_{\mathfrak{P}_{\mathcal{G} \in V_{G}^{1}(R)}} v_{\mathfrak{P}}(I) \mathfrak{P}$. Then $\mu$ is an isomorphism of Abelian groups and, for $I, J \in \underline{\operatorname{Div}}(R), I \subset J$ if and only if $\mu(I) \geq \mu(J)$ (i.e. $v_{\mathfrak{P}}(I) \geq v_{\mathfrak{P}}(J)$ for all $\mathfrak{B} \in V_{G}^{1}(R)$ ).

Let $H$ be a subgroup of $G$ such that $G / H$ is torsion.

Definition 1.19. Let $\mathfrak{p} \in V_{\mathrm{H}}^{1}\left(R^{(H)}\right)$ and $\mathfrak{P} \in V_{G}^{1}(R)$ such that $\mathfrak{P}^{(H)}=\mathfrak{p}$. We put $e_{R}(\mathfrak{p})=v_{\mathfrak{P}}\left(\mathfrak{p} R_{(\mathfrak{P})}\right)$.

REMARK 1.20. The correspondence $I \mapsto \underline{\operatorname{div}}_{R}(I R)\left(I \in \underline{\operatorname{Div}}\left(R^{(H)}\right)\right)$ defines a homomorphism $\underline{\operatorname{Div}}\left(R^{(H)}\right) \rightarrow \underline{\operatorname{Div}}(R)$ and there is the following commutative diagram of exact sequences: 


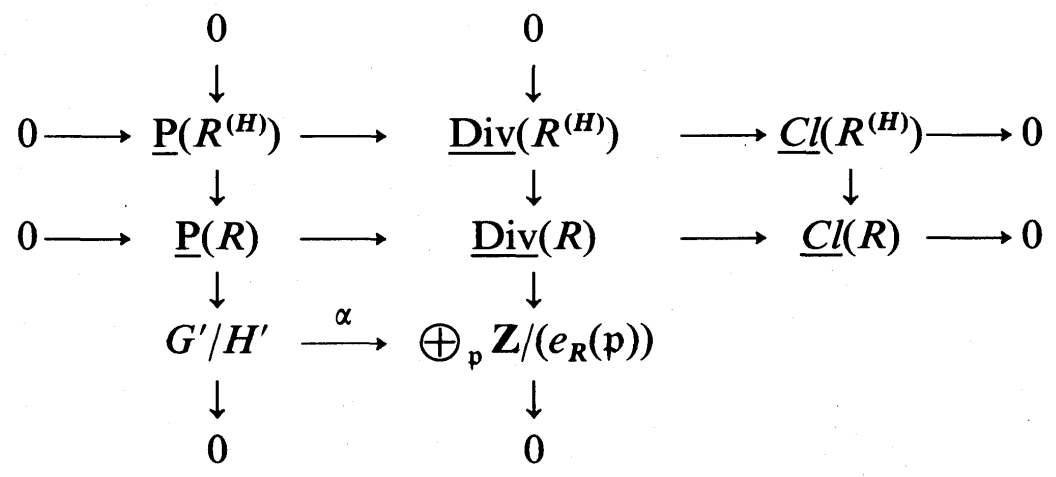

where $G^{\prime}=\left\{g \in G \mid K_{g} \neq 0\right\}, H^{\prime}$ is a subgroup of $G$ generated by $\left(G^{\prime} \cap H\right) \cup\left\{g \in G \mid R_{g}\right.$ contains a unit of $R\}$ and the map $\underline{\mathrm{P}}(R) \rightarrow G / H^{\prime}$ is defined by $x \in h(K) \mapsto$ the image of $\operatorname{deg}(x)$ in $G^{\prime} / H^{\prime}$.

EXAMPLE 1.21 (Demazure's construction of normal graded rings [4]). Let $R=\oplus_{n \geq 0} R_{n}$ be a Krull domain such that $R \neq R_{0}$ and $K=K_{0}\left[t, t^{-1}\right]$ be the homogeneous localization of $R$ at (0) with $\operatorname{deg}(t)>0$.

We put $\left\{\mathfrak{P}_{1}, \cdots \mathfrak{P}_{r}\right\}=\left\{\mathfrak{Q} \in V_{\mathbf{Z}}^{1}(R) \mid \mathfrak{Q} \not R_{+}\right\}$. Then, by (1.14), $v_{\mathfrak{P}_{i}}$ is equivalent to $v_{0}^{d_{i}, e_{i}}$ for some $d_{i}, e_{i} \in \mathbf{Z}$.

We set $X=\operatorname{Proj}(R)$ and $D=\sum_{i=1}^{r}\left(d_{i} / e_{i}\right) V_{i} \in \operatorname{Div}(X) \otimes \mathbf{Q}$ where $V_{i}$ is the prime divisor of $X$ corresponding to $\mathfrak{P}_{i}$.

Then, for $a \in K_{0}$ and $n \geq 0$,

$$
\begin{aligned}
a t^{n} \in R & \Longleftrightarrow \underline{\operatorname{div}}_{R}(a)+n \underline{\operatorname{div}}_{R}(t) \geq 0 \\
& \Longleftrightarrow \operatorname{div}_{X}(a)+n D \geq 0 \in D i v(X) \otimes \mathbf{Q} \\
& \Longleftrightarrow a \in H^{0}\left(X, \mathcal{O}_{X}(n D)\right) .
\end{aligned}
$$

(Note that if $R_{+}:=\bigoplus_{n>0} R_{n} \in V_{\mathbf{Z}}^{1}(R)$, then $v_{R_{+}}=v_{t}$.) Hence we have

$$
R \cong R(X, D):=\bigoplus_{n \geq 0} H^{0}\left(X, \mathcal{O}_{X}(n D)\right) T^{n}\left(\subset K_{0}[T]\right)
$$

We set $R(E)=\bigoplus_{n \in \mathbf{Z}} H^{0}\left(X, \mathcal{O}_{X}(E+n D)\right) T^{n}$ for $E \in \operatorname{Div}(X)$. Then, the correspondence $E \mapsto \underline{\operatorname{div}}_{R}(R(-E))(E \in \operatorname{Div}(X))$ defines a homomorphism $\operatorname{Div}(X) \rightarrow \underline{\operatorname{Div}}(R)$ and we have the following commutative diagram; 


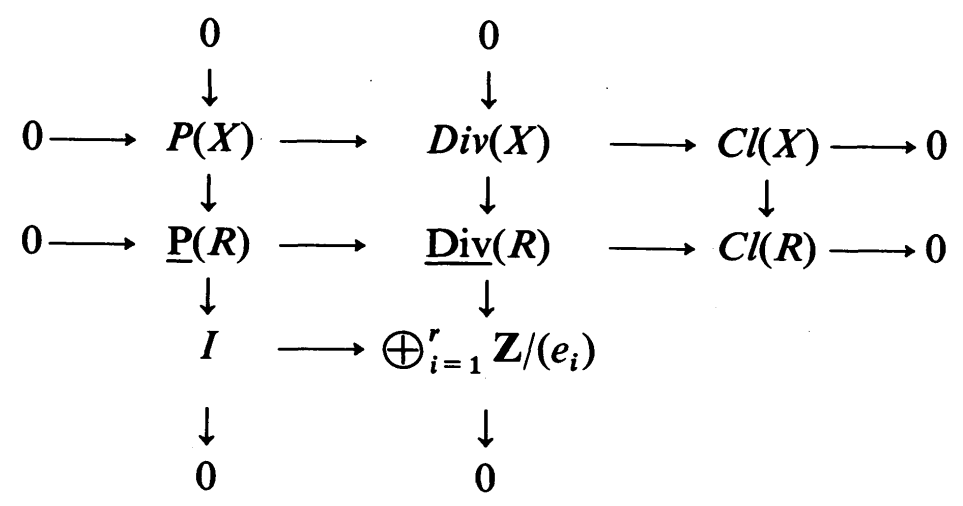

where $I \cong \mathbf{Z}$ and is generated by the image of $\underline{\operatorname{div}}_{R}(T)$. (See, for instance, Watanabe [16].)

\section{A description of $R$ from $R^{(H)}$.}

Let $R$ be a $G$-Noetherian $G$-normal $G$-domain and $K$ be the homogeneous localization of $R$ at (0). We assume that $K_{g} \neq 0$ for every $g \in G$.

Let $H$ be a subgroup of $G$ such that $G / H \cong \bigoplus_{i=1}^{r} \mathrm{Z} /\left(d_{i}\right)$ and put $A=R^{(H)}$. We put $\underline{\operatorname{Div}}(A)_{\mathbf{Q}}=\underline{\operatorname{Div}}(A) \otimes_{\mathbf{Z}} \mathbf{Q}$. For $D=\sum_{\mathfrak{p} \in V_{G}^{1}(A)} a_{\mathfrak{p}} \mathfrak{p} \in \underline{\operatorname{Div}}(A)_{\mathbf{Q}}$, we set

$$
\begin{gathered}
\operatorname{Supp}(D)=\left\{\mathfrak{p} \mid a_{\mathfrak{p}} \neq 0\right\}, \quad D(\mathfrak{p})=a_{\mathfrak{p}}, \\
{[D]=\sum_{\mathfrak{p} \in V_{G}^{1}(R)}\left[a_{\mathfrak{p}}\right] \mathfrak{p}, \quad\{D\}=D-[D],}
\end{gathered}
$$

where we denote by $A(D)$ the $H$-divisorial ideal of $A$ generated by $\left\{a \in h(K) \mid \underline{\operatorname{div}}_{A}(a)+\right.$ $D \geq 0\}$. Note that $A(D)=A([D])$ and $\underline{\operatorname{div}}_{A}(A(D))=-[D]$.

Tomari-Watanabe [14] constructed normal $\mathbf{Z}_{r}$-graded rings using an element of $\underline{\mathrm{P}}(A)_{\mathbf{Q}}$. In this section, we repeat Tomari-Watanabe's construction.

We put $K^{\prime}$ the homogeneous localization of $A$ at $(0)$. Then $K^{\prime}=K^{(H)}$ and $K=R \otimes_{A} K^{\prime}$. Furthermore, there exists $x_{i} \in h(K \backslash\{0\})(1 \leq i \leq r)$ such that $x_{i}^{d_{i}}=f_{i} \in A$ and

$$
K=\bigoplus_{\substack{1 \leq i \leq r \\ 0 \leq m_{i}<d_{i}}} K^{\prime} x_{1}^{m_{1}} x_{2}^{m_{2}} \cdots x_{r}^{m_{r}} \cong K^{\prime}\left[X_{1}, \cdots, X_{r}\right] /\left(X^{d_{1}}-f_{1}, \cdots, X^{d_{r}}-f_{r}\right)
$$

where $K^{\prime}\left[X_{1}, \cdots, X_{r}\right]$ is a polynomial ring over $K^{\prime}$ (cf. (1.6) of [9]).

We put $D_{i}=\left(1 / d_{i}\right) \underline{\operatorname{div}}_{A}\left(f_{i}\right) \in \underline{\operatorname{Div}}(A)_{\mathbf{Q}}$ for $1 \leq i \leq r$.

Proposition 2.1. We have

$$
R=\underset{\substack{1 \leq i<r \\ 0 \leq m_{i}<d_{i}}}{\bigoplus} A\left(\sum_{i=1}^{r} m_{i} D_{i}\right) \prod_{i=1}^{r} x_{i}^{m_{i}}
$$

We can give a proof in the same way as in Proposition 1.4 of Tomari-Watanabe [14].

REMARK 2.2. (1) The converse of (2.1) is also true. Let $A$ be an $H$-Noetherian $H$-normal $H$-domain and $D_{i} \in \underline{\operatorname{Div}}(A)_{\mathbf{Q}}$ such that $d_{i} D_{i}=\underline{\operatorname{div}}_{A}\left(f_{i}\right)(1 \leq i \leq r)$. Put $G=$ 
$H \oplus \mathbf{Z}^{r} / \sum_{i=1}^{r} \mathbf{Z}\left(\operatorname{deg}\left(f_{i}\right),-d_{i} e_{i}\right)$ where $e_{i}=(0, \cdots, 0, \stackrel{i}{1}, 0, \cdots, 0) \in \mathbf{Z}^{r}$. Then a $G$-graded subring

$$
R\left(A ; D_{1}, \cdots, D_{r}, f_{1}, \cdots, f_{r}\right):=\bigoplus_{\substack{1 \leq i \leq r \\ 0 \leq m_{i}<d_{i}}} A\left(\sum_{i=1}^{r} m_{i} D_{i}\right) \prod_{i=1}^{r} x_{i}^{m_{i}}
$$

of $K^{\prime}\left[X_{1}, \cdots, X_{r}\right] /\left(X_{1}^{d_{1}}-f_{1}, \cdots, X_{r}^{d_{r}}-f_{r}\right)$ is a $G$-normal $G$-domain where $K^{\prime}$ is the homogeneous localization of $A$ at (0).

(2) It is easy to verify that $e_{R}(\mathfrak{p})>1$ if and only if $D_{i}(\mathfrak{p}) \notin \mathbf{Z}$ for some $1 \leq i \leq r$. Furthermore, if we denote $D_{i}(\mathfrak{p})=a_{i} / b_{i} \in \mathbf{Q}$ with $\left(a_{i}, b_{i}\right)=1$, then $e_{R}(\mathfrak{p})$ is equal to $\operatorname{LCM}\left(\left\{b_{i} \mid a_{i} \neq 0,1 \leq i \leq r\right\}\right)$.

EXAMPLE 2.3. Let $k$ be a field and $A=k\left[s^{6}, t^{12}\right] \subset k[s, t]$ and $D_{1}=\frac{1}{2} \underline{\operatorname{div}}_{A}\left(s^{6} t^{12}\right)$, $D_{2}=\frac{1}{3} \underline{\operatorname{div}}_{A}\left(s^{6} t^{24}\right)$. Then we have

$$
R\left(A ; D_{1}, D_{2}, s^{12} t^{12}, s^{6} t^{24}\right) \cong k\left[s^{6}, s t^{10}, s^{2} t^{8}, s^{3} t^{6}, s^{4} t^{4}, s^{5} t^{2}, t^{12}\right] .
$$

For $E \in \underline{\operatorname{Div}}(A)_{\mathbf{Q}}$, we define a $G$-graded $R$-module $R\left(E ; D_{1}, \cdots, D_{r}, f_{1}, \cdots, f_{r}\right)$ as

$$
R\left(E ; D_{1}, \cdots, D_{r}, f_{1}, \cdots, f_{r}\right)=\underset{\substack{1 \leq i \leq r \\ 0 \leq m_{i}<d_{i}}}{\bigoplus} A\left(E+\sum_{i=1}^{r} m_{i} D_{i}\right) \prod_{i=1}^{r} x_{i}^{m_{i}} .
$$

Then $R\left(E ; D_{1}, \cdots, D_{r}, f_{1}, \cdots, f_{r}\right)$ is a $G$-divisorial ideal of $R$. We denote the $G$ divisorial ideal as above simply by $R(E)$, if no confusion is possible.

Proposition 2.4. We define a map

$$
\begin{aligned}
\phi: \bigoplus_{\mathfrak{p} \in V_{H_{H}}^{1}(A)} \mathbf{Z} \frac{1}{e_{R}(\mathfrak{p})} \mathfrak{p} & \longrightarrow \underline{\operatorname{Div}(R)} \\
E & \longmapsto R\left(-E ; D_{1}, \cdots, D_{r}, f_{1}, \cdots, f_{r}\right) .
\end{aligned}
$$

Then $\phi$ is an isomorphism of Abelian groups.

Proof. Since $\underline{\operatorname{div}}_{R}(R(D) R(E))=R(D+E)$ (cf. (1.17)), $\phi$ is a group homomorphism. Let $\mathfrak{P} \in V_{G}^{1}(R)$ and $\mathfrak{p}=\mathfrak{P}^{(H)}$. Then $\operatorname{div}_{R}(\mathfrak{p} R)=R(-\mathfrak{p})$ (cf. (1.17) and (1.18)). Since $\quad e_{R}(\mathfrak{p}) \underline{\operatorname{div}}_{R}(\mathfrak{P})=\underline{\operatorname{div}}_{R}(\mathfrak{p} R)=\phi(\mathfrak{p})=e_{R}(\mathfrak{p}) \phi\left(\left(1 / e_{R}(\mathfrak{p})\right) \mathfrak{p}\right) \quad($ in $\quad \underline{\operatorname{Div}}(R))$, we have $\phi\left(\left(1 / e_{R}(\mathfrak{p})\right) \mathfrak{p}\right)=\underline{\operatorname{div}}_{R}(\mathfrak{P})$ and $\phi$ is an isomorphism.

REMARK 2.5. (1) We note that $\phi\left(D_{i}\right)=\underline{\operatorname{div}}_{R}\left(R x_{i}\right)$ for $1 \leq i \leq r$ and, therefore, $\underline{\mathrm{P}}(R)$ is isomorphic to $\left\{E+\sum_{i=1}^{r} m_{i} D_{i} \mid E \in \underline{\mathrm{P}}(A), 0 \leq m_{i}<d_{i}, 1 \leq \mathrm{i} \leq \mathrm{r}\right\}$.

(2) For $0 \leq m_{i}<d_{i}(1 \leq i \leq r), A\left(\sum_{i=1}^{r} m_{i} D_{i}\right) \prod_{i=1}^{r} x_{i}^{m_{i}}$ contains a $G$-homogeneous unit of $R$ if and only if $\sum_{i=1}^{r} m_{i} D_{i} \in \underline{\mathrm{P}}(A)$.

We put $D_{R}=\sum_{\mathfrak{p} \in V_{H}(A)}\left(\left(e_{R}(\mathfrak{p})-1\right) / e_{R}(\mathfrak{p})\right) \mathfrak{p} \in \underline{\operatorname{Div}}(A)_{\mathbf{Q}}$.

LEMMA 2.6. For $E \in \underline{\operatorname{Div}}(A)$, there is the following isomorphism 


$$
\underline{\operatorname{Hom}}_{A}(R, A(E)) \cong R\left(E+D_{R} ; D_{1}, \cdots, D_{r}, f_{1}, \cdots, f_{r}\right) \text {. }
$$

ProOF. Let $0 \leq m_{i}<d_{i}(1 \leq i \leq r)$ and $g=\sum_{i=1}^{r} m_{i} \operatorname{deg}\left(x_{i}\right)$. Then, for $h \in H$, $\left[\operatorname{Hom}_{A}(R, A(E))\right]_{g+h}=\left[\operatorname{Hom}_{A}\left(R^{(-g, H)}, A(E)\right)\right]_{g+h}$ and

$$
R^{(-g, H)}=A\left(\sum_{m_{i}>0}\left(d_{i}-m_{i}\right) D_{i}\right) \prod_{m_{i}>0} x_{i}^{d_{i}-m_{i}}=A\left(\sum_{m_{i}>0}\left(\left(d_{i}-m_{i}\right) D_{i}-\underline{\operatorname{div}}_{A}\left(f_{i}\right)\right)\right) \prod_{i=1}^{r} x_{i}^{-m_{i}}
$$

Hence we have

$$
\begin{aligned}
& \underline{\operatorname{Hom}}_{A}\left(R^{(-g, H)}, A(E)\right)=\underline{\operatorname{Hom}}_{A}\left(A\left(-\sum_{i=1}^{r} m_{i} D_{i}\right) \prod_{i=1}^{r} x_{i}^{-m_{i}}, A(E)\right) \\
& \cong\left[A(E): A\left(-\sum_{i=1}^{r} m_{i} D_{i}\right)\right] \prod_{i=1}^{r} x_{i}^{m_{i}}=A\left(E-\left[-\sum_{i=1}^{r} m_{i} D_{i}\right]\right) \prod_{i=1}^{r} x_{i}^{m_{i}} \\
& =A\left(E+\left[D_{R}+\sum_{i=1}^{r} m_{i} D_{i}\right]\right) \prod_{i=1}^{r} x_{i}^{m_{i}}=A\left(E+D_{R}+\sum_{i=0}^{r} m_{i} D_{i}\right) \prod_{i=1}^{r} x_{i}^{m_{i}}
\end{aligned}
$$

and

$$
\begin{aligned}
\underline{\operatorname{Hom}}_{A}(R, A(E)) & =\underset{\substack{1 \leq i \leq r \\
0 \leq m_{i}<d_{i}}}{\bigoplus} \underline{\operatorname{Hom}}_{A}\left(A\left(-\sum_{i=1}^{r} m_{i} D_{i}\right) \prod_{i=1}^{r} x_{i}^{-m_{i}}, A(E)\right) \\
& \cong R\left(E+D_{R} ; D_{1}, \cdots, D_{r}, f_{1}, \cdots, f_{r}\right) .
\end{aligned}
$$

If $(A, \mathrm{~m})$ is $H$-local, then an $H$-canonical module $\underline{K}_{A}$ is defined as an $H$-graded $A$-module satisfying $\underline{K}_{A} \otimes_{A_{0}} \hat{A}_{0} \cong\left[H_{\hat{\mathrm{m}}}^{\mathrm{d}}(\hat{A})\right]^{\vee}$ where $\hat{A}=A \otimes_{A_{0}} \hat{A}_{\mathrm{o}}$ and $d=\underline{\operatorname{dim}}(A)$ (cf. section 3 of [9]). Furthermore, if $A$ is $H$-normal and $\underline{K}_{A}$ exists, then $\underline{K}_{A}$ is $H$-divisorial. We denote by $\boldsymbol{\Omega}_{A} \in \underline{\operatorname{Div}}(A)$ the $H$-divisor satisfying $\underline{K}_{A}=A\left(\Omega_{A}\right)$, if $\underline{K}_{A}$ exists.

As a direct consequence of (2.6), we have the following:

Proposition 2.7 (Theorem 3.2 of Tomari-Watanabe [14]). Assume that $A$ is $H$-local and has an H-canonical module $\underline{K}_{A}=\underline{\operatorname{div}}_{A}\left(\Omega_{A}\right)$.

(1) $\underline{K}_{R}=R\left(\Omega_{A}+D_{R} ; D_{1}, \cdots, D_{r}, f_{1}, \cdots, f_{r}\right)$.

(2) $\underline{K}_{R}$ is free if and only if $\Omega_{A}+D_{R}-\sum_{i=1}^{r} m_{i} D_{i} \in \underline{\mathrm{P}}(A)$ for some $0 \leq m_{i}<d_{i}$ $(1 \leq i \leq r)$.

\section{Complete intersections.}

We keep notations as in section 2. Suppose that $R$ has a unique $G$-maximal ideal $\mathfrak{M}$. We put $\mathfrak{m}=\mathfrak{M}^{(H)} \subset A$ and assume that $A / \mathfrak{m}=A_{0} / \mathfrak{m}_{0}$.

In this section, we consider a condition for $R$ to be (locally) a complete intersection under the following assumptions. 
Assumption 3.1. (1) $A$ is a factorial domain and locally a complete intersection.

(2) $\quad R / \mathfrak{M}=A / \mathfrak{m}\left(=A_{0} / \mathrm{m}_{0}\right)\left(\right.$ or $\sum_{i=1}^{r} m_{i} D_{i} \notin \underline{\mathrm{P}}(A)$ for $\left.\left(m_{1}, \cdots, m_{r}\right) \neq(0, \cdots, 0)\right)$.

Throughout this section, we assume the above conditions (1) and (2).

We set $\operatorname{Deg}(R)=\left\{\left\{\sum_{i=1}^{r} m_{i} D_{i}\right\} \mid 0 \leq m_{i}<d_{i}(1 \leq i \leq r)\right\}$ and denote by $\operatorname{Fund}(R)$ the set of minimal elements of $\operatorname{Deg}(R)$ with respect to the order of $\underline{\operatorname{Div}}(A)_{\mathbf{Q}}$.

Since $\left[\sum_{i=1}^{r} m_{i} D_{i}\right] \in \underline{\mathrm{P}}(A),\left\{\sum_{i=1}^{r} m_{i} D_{i}\right\}$ is a principal ideal of $R$ and $\operatorname{Deg}(R) \subset \underline{\mathrm{P}}(R)$. For $E \in \operatorname{Fund}(R)$, we put $y_{E} \in h(R)$ a homogeneous element satisfying $\operatorname{div}_{R}\left(y_{E}\right)=E$. Also, by (3.1), $\alpha: G / H \rightarrow \bigoplus_{\mathfrak{p} \in V_{H}^{1}(A)} Z /\left(e_{R}(\mathfrak{p})\right)$ is injective where $\alpha$ is as in (1.20).

Put $\Lambda=\left\{\mathfrak{p} \in V_{H}^{1}(A) \mid e_{R}(\mathfrak{p})>1\right\}$. For $\Lambda^{\prime} \subset \Lambda$, we denote by $G_{\Lambda^{\prime}}$ a subgroup of $G$ such that $G_{\Lambda^{\prime}} \supset H$ and $G_{\Lambda^{\prime}} / H=\alpha^{-1}\left(\bigoplus_{\mathfrak{p} \in \Lambda^{\prime}} \mathbf{Z} /\left(e_{R}(\mathfrak{p})\right)\right)$.

LEMMA 3.2. (1) For $E \in \underline{\mathrm{P}}(R)$ with $E>0$ and $E \notin \underline{\mathrm{P}}(A)$, there exists $D \in \operatorname{Fund}(R)$ such that $D \leq E$ (i.e. Fund $(R)$ is the set of minimal elements of $\{E \in \underline{\mathrm{P}}(R) \mid E>0$ and $E \notin \underline{\mathrm{P}}(A)\})$.

(2) $R=A\left[y_{E} \mid E \in \operatorname{Fund}(R)\right]$.

$\left(2^{\prime}\right)$ Every element of $\operatorname{Deg}(R)$ is a sum of elements of $\operatorname{Fund}(R)$.

(3) $\operatorname{Fund}\left(R^{\left.G_{\Lambda^{\prime}}\right)}\right)=\left\{E \in \operatorname{Fund}(R) \mid \operatorname{Supp}(E) \subset \Lambda^{\prime}\right\}$ for $\Lambda^{\prime} \subset \Lambda$.

Proof. (1) For every $E \in \underline{\mathrm{P}}(R) \backslash \underline{\mathrm{P}}(A)$ with $E>0$, there exist $0 \leq m_{i}<d_{i}(1 \leq i \leq r)$ and $E^{\prime} \in \underline{\mathrm{P}}(A)$ such that $E=E^{\prime}+\sum_{i=1}^{r} m_{i} D_{i}$ and $E^{\prime}+\left[\sum_{i=1}^{r} m_{i} D_{i}\right] \geq 0$ (cf. (1) of (2.5)). Hence $E \geq\left\{\sum_{i=1}^{r} m_{i} D_{i}\right\}$. This implies the assertion (1).

(2) Let $D \in \underline{\mathrm{P}}(R)$ such that $D>0$ and $D \notin \underline{\mathrm{P}}(A)$. Then there exists $E_{1} \in \operatorname{Fund}(R)$ such that $D \geq E_{1}$ by (1). Since $0 \leq D-E_{1} \in \underline{\mathrm{P}}(R)$, if $D-E_{1} \notin \underline{\mathrm{P}}(A)$, then there exists $E_{2} \in \operatorname{Fund}(R)$ such that $D-E_{1} \geq E_{2}$. Continuing this process, we can write $D=$ $\underline{\operatorname{div}}_{A}(a)+\sum_{i=1}^{s} p_{i} E_{i}$ where $a \in h(A), E_{i} \in \operatorname{Fund}(R)$ and $p_{i}$ 's are non-negative integers. This implies that $R$ is generated by $\left\{y_{E} \mid E \in \operatorname{Fund}(R)\right\}$ as an $A$-algebra.

(3) For $y \in h(R), \operatorname{deg}(y) \in G_{\Lambda^{\prime}}$ if and only if $\operatorname{Supp}\left(\left\{\underline{\operatorname{div}}_{R}(y)\right\}\right) \subset \Lambda^{\prime}$ by (1.20). Hence, by $(1), \operatorname{Fund}\left(R^{\left(G_{\Lambda^{\prime}}\right)}\right)$ is the set of minimal elements of $\{E \in \underline{\mathrm{P}}(R) \mid[E] \neq E, E>0$ and $\left.\operatorname{Supp}(\{E\}) \subset \Lambda^{\prime}\right\}$ and coincide with $\left\{E \in \operatorname{Fund}(R) \mid \operatorname{Supp}(E) \subset \Lambda^{\prime}\right\}$.

For $\Lambda^{\prime} \subset \Lambda$, we define a polynomial ring $S_{\Lambda^{\prime}}$ by $S_{\Lambda^{\prime}}=A\left[Y_{E} \mid E \in \operatorname{Fund}\left(R^{\left(G_{A^{\prime}}\right)}\right)\right]$ and $M_{A^{\prime}}=\mathfrak{m} S_{\Lambda^{\prime}}+\left(Y_{E} \mid E \in \operatorname{Fund}\left(R^{\left(G_{\Lambda^{\prime}}\right)}\right)\right)$. We define a surjective $A$-algebra homomorphism

$$
\psi_{\Lambda^{\prime}}: S_{\Lambda^{\prime}} \rightarrow R^{\left(G_{\Lambda^{\prime}}\right)} \quad \text { by } \quad \psi_{\Lambda^{\prime}}\left(Y_{E}\right)=y_{E}
$$

for $E \in \operatorname{Fund}\left(R^{\left(G_{\Lambda^{\prime}}\right)}\right)$ and put $J_{\Lambda^{\prime}}=\operatorname{ker}\left(\psi_{\Lambda^{\prime}}\right)$. It is easy to verify that $J_{\Lambda^{\prime}}$ is generated by elements of the form $Y^{\alpha}-a Y^{\beta}(\neq 0)\left(a \in h(A), Y^{\alpha}, Y^{\beta}\right.$ are monomials of $\left.S_{\Lambda^{\prime}}\right)$ and $J_{\Lambda^{\prime}}=J_{A} \cap S_{A^{\prime}}$.

For $E \in \operatorname{Fund}(R)$, there exists an element of $J_{A}$ of the form $Y_{E}^{d}-a Y^{\beta}$, where $a \in h(A)$ and $Y^{\beta}$ is a monomial of $S_{A}$, since $G / H$ is torsion. Put $d_{E}=\inf \left\{d \mid 0 \neq Y_{E}^{d}-a Y^{\beta} \in J_{A}\right\}$. We fix an element

$$
F(E)=Y_{E}^{d_{E}}-a_{E} Y^{\beta_{E}} \in J_{A}
$$


For $\Lambda^{\prime} \subset \Lambda$, if $\operatorname{Supp}(E) \subset \Lambda^{\prime}$, then $F(E) \in J_{\Lambda^{\prime}}$. Moreover, $\left\{F(E) \mid E \in \operatorname{Fund}\left(R^{\left(G_{\Lambda^{\prime}}\right)}\right)\right\}$ is a part of minimal basis of $\left(J_{\Lambda^{\prime}}\right)_{M_{\Lambda^{\prime}}}$.

Proposition 3.3. For $\Lambda^{\prime} \subset \Lambda, R^{\left(G_{\Lambda^{\prime}}\right)}$ is locally a complete intersection if and only if $J_{A^{\prime}}$ is generated by $\left\{F(E) \mid E \in \operatorname{Fund}\left(R^{\left(G_{A^{\prime}}\right)}\right)\right\}$.

To prove Proposition 3.3, we need some preliminaries.

For $\Lambda^{\prime} \subset \Lambda$, we define a directed graph $\mathscr{G}_{\Lambda^{\prime}}$ of a vertex set $V\left(\mathscr{G}_{\Lambda^{\prime}}\right)=\left\{Y_{E} \mid E \epsilon\right.$ Fund $\left.\left(R^{\left(G_{A^{\prime}}\right)}\right)\right\}$ and directed edges $D E\left(\mathscr{G}_{\Lambda^{\prime}}\right)$ as follows: an ordered pair $\left(Y_{E}, Y_{D}\right)$ is in $D E\left(\mathscr{G}_{A^{\prime}}\right)$, if $Y_{D}$ divides $Y^{\beta_{E}}$ where $Y^{\beta_{E}}$ is as above.

LEMMA 3.4. ((3.5) of Nakajima [10] and (2.1) of Eto [5]). Suppose that $\left(J_{A^{\prime}}\right)_{M_{A^{\prime}}}$ is generated by $\left\{F(E) \mid\right.$ Fund $\left.\left(R^{\left(G_{A^{\prime}}\right)}\right)\right\}$.

(1) There exists a linear ordering $\leq_{\Lambda^{\prime}}$ on $V\left(\mathscr{G}_{\Lambda^{\prime}}\right)$ such that $Y_{E} \geq_{A^{\prime}} Y_{D}$, if $\left(Y_{E}, Y_{D}\right) \in$ $D E\left(\mathscr{G}_{\Lambda}\right)$.

(2) $\left\{F(E) \mid E \in\right.$ Fund $\left.\left(R^{\left(G_{A^{\prime}}\right)}\right)\right\}$ forms a regular sequence (in any order).

Proof. (1) We have only to show that $\mathscr{G}_{\Lambda^{\prime}}$ has no cycle. Therefore, we assume the contrary. Then there exist $\left(Y_{E_{1}}, Y_{E_{2}}\right),\left(Y_{E_{2}}, Y_{E_{3}}\right), \cdots,\left(Y_{E_{k-1}}, Y_{E_{k}}\right),\left(Y_{E_{k}}, Y_{E_{1}}\right) \in$ $D E\left(\mathscr{G}_{\Lambda^{\prime}}\right)$. We may assume that $E_{i} \neq E_{j}$ for $i \neq j$. Then, by definition of $D E\left(\mathscr{G}_{A^{\prime}}\right)$, $\left\{F\left(E_{1}\right), \cdots, F\left(E_{k}\right)\right\}$ is contained in $\left(Y_{E_{1}}, \cdots, Y_{E_{k}}\right)$. Since $\left(J_{\Lambda^{\prime}}\right)_{M_{A^{\prime}}}$ is generated by $\left\{F(E) \mid E \in \operatorname{Fund}\left(R^{\left(G_{A^{\prime}}\right)}\right)\right\}$, the number of minimal generators of $\left(J_{A^{\prime}}\right)_{M_{A^{\prime}}}+\left(Y_{E_{1}}, \cdots\right.$, $\left.Y_{E_{k}}\right)_{M_{\Lambda^{\prime}}}$ is at most $\left|\operatorname{Fund}\left(R^{\left(G_{A^{\prime}}\right)}\right)\right|-k+k=\left|\operatorname{Fund}\left(R^{\left(G_{A^{\prime}}\right)}\right)\right|$. On the other hand, since $R^{\left(G_{A^{\prime}}\right)}$ is $G_{\Lambda^{\prime}}$-domain, $Y_{E_{1}}$ is not a zero divisor of $S_{A^{\prime}} / J_{A^{\prime}}$ and

$$
\left.\left|\operatorname{Fund}\left(R^{\left(G_{\Lambda^{\prime}}\right)}\right)\right|=h t\left(\left(J_{A^{\prime}}\right)_{M_{A^{\prime}}}\right)<h t\left(\left(J_{\Lambda^{\prime}}, Y_{E_{1}}, \cdots, Y_{E_{k}}\right)_{M_{A^{\prime}}}\right)\right) \text {. }
$$

This is a contradiction. Hence $\mathscr{G}_{\Lambda^{\prime}}$ has no cycle.

(2) We put $J^{\prime}=\left(\left\{F(E) \mid E \in \operatorname{Fund}\left(R^{\left(G_{\Lambda^{\prime}}\right)}\right)\right\}\right)$ and extend $\leq_{\Lambda^{\prime}}$ to a monomial ordering of $S_{\Lambda^{\prime}}$, lexicographically. Then, for $E \in \operatorname{Fund}\left(R^{\left(G_{A^{\prime}}\right)}\right), \quad Y_{E}^{d_{E}}>_{A^{\prime}} Y^{\beta_{E}}$ and $\left\{F(E) \mid E \in\right.$ Fund $\left.\left(R^{\left(G_{A^{\prime}}\right)}\right)\right\}$ forms a Gröbner basis of $J^{\prime}$ (cf. Proposition 9.2(b) of [13]). In particular, the initial term $\operatorname{in}\left(J^{\prime}\right)$ of $J^{\prime}$ is generated by $\left\{Y_{E}^{d_{E}} \mid E \in \operatorname{Fund}\left(R^{\left(G_{A^{\prime}}\right)}\right)\right\}$. By standard arguments of the theory of Gröbner basis, this implies $\left\{F(E) \mid E \in \operatorname{Fund}\left(R^{\left(G_{A^{\prime}}\right)}\right)\right\}$ is a regular sequence. (See for instance [13].)

Proof of (3.3). The "if" part follows from (3.4). We shall show the "only if" part.

We put $S=S_{A^{\prime}}, M=M_{A^{\prime}}$, and $J=J_{A^{\prime}}$. Since $R^{\left(G_{A^{\prime}}\right)}$ is $A$-free, we have $J / \mathrm{m} J \cong$ $\operatorname{ker}\left(\psi_{A^{\prime}} \otimes_{A} A / \mathrm{m}\right)$. Thus the number $\mu\left(J_{M}\right)$ of minimal generators of $J_{M}$ is equal to the number $\mu\left(J_{M}+\mathrm{m} S_{M} / \mathfrak{m} S_{M}\right)$. Then, since $\left(R^{\left(G_{\Lambda^{\prime}}\right)}\right)_{M}$ is complete intersection, $\mu\left(J_{M}\right)=$ $\left|\operatorname{Fund}\left(R^{G_{\Lambda^{\prime}}}\right)\right|\left(\right.$ cf. Theorem 2 of [2]). Namely, if we put $J^{\prime}=\left(\left\{F(E) \mid E \in \operatorname{Fund}\left(R^{\left(G_{A^{\prime}}\right)}\right)\right\}\right)$, then $J_{M}=J_{M}^{\prime}$ since $\left\{F(E) \mid E \in\right.$ Fund $\left.\left(R^{\left(G_{A^{\prime}}\right)}\right)\right\}$ is part of minimal generators of $J_{M}$. Thus $J=J^{\prime}+M J$ and $J=J^{\prime}+M^{n} J$ for every $n>0$. For $E \in \operatorname{Fund}\left(R^{\left(G_{A^{\prime}}\right)}\right)$, we put $d=$ $\prod_{Y_{D} \leq A^{\prime} Y_{E}} d_{D}$ where $<_{A^{\prime}}$ is as in (1) of (3.4). By (2) of (3.1), there exists $D \in \operatorname{Fund}\left(R^{\left(G_{A^{\prime}}\right)}\right)$ 
such that $Y_{D}<_{A^{\prime}} Y_{E}$ and $F(D)=Y_{D}^{d_{D}}-a_{D}$ for some $a_{D} \in \mathfrak{m}$. This implies that $Y_{E}^{d} \in J^{\prime}+\mathfrak{m} S$. Hence, for $n \gg 0, M_{\Lambda^{\prime}}^{n} J \subset J^{\prime}+\mathrm{m} S$ and $J+\mathrm{m} S / \mathrm{m} S=J^{\prime}+\mathrm{m} S / \mathrm{m} S$ (or $S / J^{\prime}+\mathrm{m} S \cong$ $\left.\left(S / J^{\prime}\right) \otimes_{A} A / \mathrm{m} \cong R^{\left(G_{A^{\prime}}\right)} \otimes_{A} A / \mathrm{m}\right)$. On the other hand, $S / J^{\prime}$ is $A$-free, by the proof of (2) of (3.4). Then $\operatorname{rank}_{A}\left(S / J^{\prime}\right)=\operatorname{dim}_{A / \mathrm{m}}\left(\left(S / J^{\prime}\right) \otimes_{A} A / \mathrm{m}\right)=\operatorname{dim}_{A / \mathfrak{m}}\left(R^{\left(G_{A^{\prime}}\right)} \otimes_{A} A / \mathrm{m}\right)=$ $\operatorname{rank}_{A}\left(R^{\left(G_{\Lambda^{\prime}}\right)}\right)$ and the canonical surjection $S / J^{\prime} \rightarrow R^{\left(G_{A^{\prime}}\right)}$ is isomorphism. Hence we have $J=J^{\prime}$.

COROLlARY 3.5. If $R$ is locally a complete intersection, then so is $R^{\left(G_{\Lambda^{\prime}}\right)}$ for any $\Lambda^{\prime} \subset \Lambda$.

Proof. Suppose that $R$ is locally a complete intersection. Then, by (3.3), $J_{A}$ is generated by $\{F(E) \mid E \in$ Fund $(R)\}$. Also, by the proof of (3.4), $J_{\Lambda^{\prime}}=J_{A^{\prime}} \cap S_{A^{\prime}}$ is generated by $\left\{F(E) \mid E \in \operatorname{Fund}(R), F(E) \in S_{\Lambda^{\prime}}\right\}=\left\{F(E) \mid E \in \operatorname{Fund}\left(R^{\left(G_{\Lambda^{\prime}}\right)}\right)\right\}$ for $\Lambda^{\prime} \subset \Lambda$. Thus $R^{\left(G_{A^{\prime}}\right)}$ is locally a complete intersection for $\Lambda^{\prime} \subset \Lambda$.

DEFINITION 3.6. Let $\Gamma$ be a finite subset of $\underline{\operatorname{Div}}(A)_{\mathbf{Q}} \backslash\{0\}$ and $w: \Gamma \rightarrow \mathbf{N}_{+}$be a map. Here we denote by $\mathbf{N}_{+}$the set of all positive integers. We call that $(\Gamma, w)$ is a datum, if it satisfies the following conditions;

(1) for $D, E \in \Gamma(D \neq E)$, one of the following cases occurs;

(a) $\operatorname{Supp}(D) \subsetneq \operatorname{Supp}(E)$, (b) $\operatorname{Supp}(D) \supsetneq \operatorname{Supp}(E)$, (c) $\operatorname{Supp}(D) \bigcap \operatorname{Supp}(E)=\varnothing$,

(2) for $E \in \Gamma$, there is a relation $w(E) E=\sum_{i=1}^{k} E_{i}+\sum_{j=1}^{l} \mathfrak{p}_{j}$ where $\left\{E_{1}, \cdots, E_{k}\right\}=$ $\{D \in \Gamma \mid D \prec E\}$ and $\left\{\mathfrak{p}_{1}, \cdots, \mathfrak{p}_{l}\right\}=\operatorname{Supp}(E) \backslash \bigcup_{i=1}^{k} \operatorname{Supp}\left(E_{i}\right)$. (We write $D \prec E$ if $\operatorname{Supp}(D) \subsetneq \operatorname{Supp}(E)$ and there is no element $D^{\prime} \in \Gamma$ such that $\operatorname{Supp}(D) \subsetneq \operatorname{Supp}\left(D^{\prime}\right) \subsetneq$ $\operatorname{Supp}(E)$.)

Let $(\Gamma, w)$ be a datum. For $E \in \Gamma$ and $\mathfrak{p} \in \operatorname{Supp}(E)$, we set

$$
\begin{gathered}
\Gamma_{E, \mathfrak{p}}=\{D \in \Gamma \mid \mathfrak{p} \in \operatorname{Supp}(D) \subset \operatorname{Supp}(E)\}, \\
e_{E}(\mathfrak{p})=\prod_{D \in \Gamma_{E, \mathfrak{p}}} w(D) .
\end{gathered}
$$

Then we have $E=\sum_{\mathfrak{p} \in \operatorname{Supp}(E)}\left(1 / e_{E}(\mathfrak{p})\right) \mathfrak{p}$.

DEFINITION 3.7. Let $(\Gamma, w)$ be a datum.

(1) Let $A\left[Y_{E} \mid E \in \Gamma\right]$ be a polynomial ring over $A$. We put $a_{E} \in h(A)$ a homogeneous element satisfying $\underline{\operatorname{div}}_{A}\left(a_{E}\right)=w(E) E-\sum_{D \prec E} D$ for $E \in \Gamma$ and set

$$
R(\Gamma)=A\left[Y_{E} \mid E \in \Gamma\right] /\left(Y_{E}^{w(E)}-a_{E} \prod_{D \prec E} Y_{D} \mid E \in \Gamma\right) .
$$

(2) We put $G(\varnothing)=H$ and define an Abelian group $G(\Gamma)$ such that $R(\Gamma)$ is a $G(\Gamma)$-graded ring by

$$
G(\Gamma)=\mathbf{Z}^{(\Gamma)} /\left\langle w(E) \mathbf{e}_{E}-\sum_{D \prec E} \mathbf{e}_{D} \mid E \in \Gamma\right\rangle,
$$


where $\mathbf{Z}^{(\Gamma)}=\bigoplus_{E \in \Gamma} \mathbf{Z e _ { E }}$ is a free abelian group with free basis $\left\{\mathbf{e}_{E} \mid E \in \operatorname{Fund}(R)\right\}$.

(3) Let $\left\{E_{1}, \cdots, E_{l}\right\}=\{E \in \Gamma \mid \operatorname{Supp}(E)$ is maximal in $\{\operatorname{Supp}(D) \mid D \in \Gamma\}\}$ and $\mathfrak{p} \in$ $V_{H}^{1}(A)$. We put

$$
e(\mathfrak{p})=\left\{\begin{array}{cl}
e_{E_{i}}(\mathfrak{p}) & \text { if } \mathfrak{p} \in \operatorname{Supp}\left(E_{i}\right) \text { for some } i \\
1 & \text { otherwise }
\end{array}\right.
$$

EXAMPLe 3.8. Let $k$ be a field. We put $A=k[[a, b, c, d]]$ the formal power series ring and

$$
\begin{gathered}
E_{1}=\frac{1}{12} \operatorname{div}_{A}(a c)+\frac{1}{8} \operatorname{div}_{A}(b d), \\
E_{2}=\frac{1}{6} \operatorname{div}_{A}(a)+\frac{1}{4} \operatorname{div}_{A}(b), \quad E_{3}=\frac{1}{6} \operatorname{div}_{A}(c)+\frac{1}{4} \operatorname{div}_{A}(d), \\
E_{4}=\frac{1}{3} \operatorname{div}_{A}(a), \quad E_{5}=\frac{1}{2} \operatorname{div}_{A}(b), \quad E_{6}=\frac{1}{3} \operatorname{div}_{A}(c), \quad E_{7}=\frac{1}{2} \operatorname{div}_{A}(d),
\end{gathered}
$$

and $\Gamma=\left\{E_{1}, \cdots, E_{7}\right\}$. We define the map $w: \Gamma \rightarrow \mathbf{N}_{+}$by $w\left(E_{1}\right)=w\left(E_{2}\right)=w\left(E_{3}\right)=w\left(E_{5}\right)=$ $w\left(E_{7}\right)=2$ and $w\left(E_{4}\right)=w\left(E_{6}\right)=3$. Then $(\Gamma, w)$ is a datum and

$$
R(\Gamma)=A\left[Y_{E_{1}}, \cdots, Y_{E_{7}}\right] / J
$$

where $J=\left(Y_{E_{1}}^{2}-Y_{E_{2}} Y_{E_{3}}, Y_{E_{2}}^{2}-Y_{E_{4}} Y_{E_{5}}, Y_{E_{3}}^{2}-Y_{E_{6}} Y_{E_{7}}, Y_{E_{4}}^{3}-a, Y_{E_{5}}^{2}-b, Y_{E_{6}}^{3}-c, Y_{E_{7}}^{2}-d\right)$.

Proposition 3.9. Let $(\Gamma, w)$ be a datum. Then the following hold.

(1) $R(\Gamma)$ is $G(\Gamma)$-normal and $\underline{\operatorname{Div}}(R(\Gamma)) \cong \bigoplus_{\mathfrak{p} \in V_{H}^{1}(A)} \mathbf{Z}(1 / e(\mathfrak{p})) \mathfrak{p}$.

(2) $\operatorname{div}_{R}\left(Y_{E} R(\Gamma)\right)=E$ for $E \in \Gamma$ by the identification as in (2.4).

(3) $\operatorname{Fund}(R(\Gamma))=\Gamma$.

The above proposition follows from the next remark.

REMARK 3.10. Let $B$ be a $G^{\prime}$-normal and $a \in h(B)$. We put $G^{\prime \prime}=G^{\prime} \oplus \mathbf{Z} /\langle(\operatorname{deg}(a)$, $-n)\rangle$. Then, by (2.1) and (2.2), $B^{\prime}:=B[X] /\left(X^{n}-a\right)$ is $G^{\prime \prime}$-normal if and only if $\underline{\operatorname{div}}_{B}(a)(\mathfrak{q}) \leq 1$ for every $\mathfrak{q} \in V_{G^{\prime}}^{1}(B)$. In this case, $\underline{\operatorname{Div}}\left(B^{\prime}\right)=\oplus_{\mathfrak{q} \in V_{G}^{1},(B)} \mathbf{Z}(1 / e(\mathfrak{q})) \mathfrak{q}$ and $\underline{\operatorname{div}}_{B^{\prime}}\left(X B^{\prime}\right)=(1 / n) \underline{\operatorname{div}}_{B}(a)$ where $e(q)=n($ resp. $e(q)=1)$, if $\left.\mathfrak{q} \in \operatorname{Supp}^{\prime} \underline{\operatorname{div}}_{B}(a)\right)$ (resp. $q \notin$ $\left.\operatorname{Supp}\left(\operatorname{div}_{B}(a)\right)\right)$ (cf. (2.4)).

We state our main result as follows.

THEOREM 3.11. The following conditions are equivalent.

(1) $R$ is locally a complete intersection.

(2) There exists a datum $(\Gamma, w)$ such that $G \cong G(\Gamma)$ and $R \cong R(\Gamma)$.

The proof of (2) $\Rightarrow(1)$ of (3.11) is easy. Therefore, it is enough to show the following proposition.

Proposition 3.12. Suppose that $R$ is locally a complete intersection. We put $\Gamma=\operatorname{Fund}(R)$. Then the following hold.

(1) There exists a map $w: \Gamma \rightarrow \mathbf{N}_{+}$such that $(\Gamma, w)$ is a datum. 
(2) $R(\Gamma)=S_{\Lambda} / J_{\Lambda}(\cong R)$.

(3) $G \cong G(\Gamma)$.

Proof. (1) We prove the assertion by induction on $|\Lambda|$. If $\Lambda=\varnothing$, then $R=A$ and $\operatorname{Fund}(R)=\varnothing$. We assume that $\Lambda \neq \varnothing$. Then, by (3.5), $R^{\left(G_{\Lambda^{\prime}}\right)}$ is locally a complete intersection and, by induction hypothesis, Fund $\left(R^{\left(G_{\Lambda^{\prime}}\right)}\right)$ is a datum for all $\Lambda^{\prime} \subsetneq \Lambda$. Since $A$ is factorial, $\sum_{\mathfrak{p} \in A} \mathfrak{p} \in \underline{\mathrm{P}}(A)$ and, by (2) of (2.7), $D:=\sum_{\mathfrak{p} \in \Lambda}\left(1 / e_{R}(\mathfrak{p})\right) \mathfrak{p} \in \operatorname{Deg}(R)$. The proof is divided into two cases: (1) $D \notin \operatorname{Fund}(R)$, (2) $D \in \operatorname{Fund}(R)$.

Case (1). We can write $D=E_{1}+\cdots+E_{p}$ for $E_{1}, \cdots, E_{p} \in \operatorname{Fund}(R)(p \geq 2)$ by (3.2), (2'). Then $\operatorname{Supp}\left(E_{i}\right) \cap \operatorname{Supp}\left(E_{j}\right)=\varnothing$ for $i \neq j$. We put $\Lambda_{i}=\operatorname{Supp}\left(E_{i}\right)$ for $1 \leq i \leq p$.

Let $E^{\prime} \in \operatorname{Fund}(R)$. If $\Lambda_{i} \subset \operatorname{Supp}\left(E^{\prime}\right)$, then $E_{i} \leq E^{\prime}$ and, since $E^{\prime} \in \operatorname{Fund}(R)$, we have $E_{i}=E^{\prime}$. We assume that $\Lambda_{i} \not \operatorname{Supp}\left(E^{\prime}\right)$ for $i=1, \cdots, p$. Since $\operatorname{Supp}\left(E^{\prime}\right) \subset \Lambda$, $\operatorname{Supp}\left(E_{j}\right) \cap \operatorname{Supp}\left(E^{\prime}\right) \neq \varnothing$ for some $j$. Then $\Lambda^{\prime}:=\Lambda_{j} \cup \operatorname{Supp}\left(E^{\prime}\right) \subsetneq \Lambda$ since $\Lambda_{i} \not \operatorname{Supp}\left(E^{\prime}\right)$ for all $i$ and $\Lambda_{i} \cap \Lambda_{k}=\varnothing$ for $i \neq k$. By induction hypothesis and the fact that $E_{j}, E^{\prime} \in \operatorname{Fund}\left(R^{\left(G_{A^{\prime}}\right)}\right)$ (cf. (3.2)), we have $\operatorname{Supp}\left(E^{\prime}\right) \subset \operatorname{Supp}\left(E_{j}\right)$. Hence Fund $(R)$ is disjoint union of Fund $\left(R^{\left(G_{A i}\right)}\right)(1 \leq i \leq p)$. On the other hand, Fund $\left(R^{\left(G_{A i}\right)}\right)$ is a datum $(1 \leq i \leq p)$, by induction hypothesis. Thus Fund $(R)$ is also a datum.

Case (2) $D \in \operatorname{Fund}(R)$. We put $\left\{E_{1}, \cdots, E_{p}\right\}=\{E \in \operatorname{Fund}(R) \mid E \prec D\}$.

Claim 1. $\operatorname{Supp}\left(E_{i}\right) \cap \operatorname{Supp}\left(E_{j}\right)=\varnothing$ for $i \neq j$.

Assume that $\operatorname{Supp}\left(E_{i}\right) \cap \operatorname{Supp}\left(E_{j}\right)$ is not empty for some $i \neq j$. Then we have that $\Lambda=\operatorname{Supp}\left(E_{i}\right) \cup \operatorname{Supp}\left(E_{j}\right)$, since Fund $\left(R^{\left(G_{\Lambda^{\prime}}\right)}\right)$ is a datum for any $\Lambda^{\prime} \subsetneq \Lambda$. Since $E_{i}+E_{j} \geq D$, we have a relation $E_{i}+E_{j}=n D+E$ where $0 \leq E \in \underline{\mathrm{P}}(R)$ and $\operatorname{Supp}(E) \subsetneq \Lambda$. In other words, $Y_{E_{i}} Y_{E_{j}}-a Y_{D}^{n} Y^{\beta} \in J_{A}$ where $a \in h(A)$ such that $\operatorname{div}_{R}\left(a \psi_{A}\left(Y^{\beta}\right)\right)=E$. Then, by (3.3), there exists $E^{\prime} \in \operatorname{Fund}(R)$ such that $Y^{\beta_{E^{\prime}}}$ divides $Y_{E_{i}} Y_{E_{j}}$ and $a_{E^{i}}$ is unit where $a_{E^{\prime}} Y^{\beta_{E^{\prime}}}$ is the term of the polynomial $F\left(E^{\prime}\right) \in S_{\Lambda}$. Since $E_{i}, E_{j} \in \operatorname{Fund}(R)$, we have $Y^{\beta_{E^{\prime}}}=Y_{E_{i}} Y_{E_{j}}$ and $F\left(E^{\prime}\right)=Y_{E_{i}}^{d_{E_{i}}}-a_{E^{\prime}} Y_{E_{i}} Y_{E_{j}}$. Also, since $\operatorname{Supp}\left(E^{\prime}\right) \supsetneq \operatorname{Supp}\left(E_{i}\right)$, we have $E^{\prime}=D$. Namely, $d_{D} D=E_{i}+E_{j}$. On the other hand, if there exists $1 \leq k \leq p, k \neq i, j$, then $\operatorname{Supp}\left(E_{i}\right) \cup$ $\operatorname{Supp}\left(E_{k}\right)=\Lambda$ by induction hypothesis. Then $E_{i}+E_{k}=d_{D} D$ and $E_{k}=E_{j}$. This is a contradiction. Hence we have $p=2$.

We put $\operatorname{Supp}\left(E_{1}\right) \backslash \operatorname{Supp}\left(E_{2}\right)=\left\{\mathfrak{p}_{1}, \cdots, \mathfrak{p}_{s}\right\}, \operatorname{Supp}\left(E_{1}\right) \cap \operatorname{Supp}\left(E_{2}\right)=\left\{\mathfrak{p}_{s+1}, \cdots, \mathfrak{p}_{t}\right\}$ and $\operatorname{Supp}\left(E_{2}\right) \backslash \operatorname{Supp}\left(E_{1}\right)=\left\{\mathfrak{p}_{t+1}, \cdots, \mathfrak{p}_{m}\right\}$. Since $d_{D} D=E_{1}+E_{2}$, we can write

$$
E_{1}=\sum_{i=1}^{s} \frac{d_{D}}{e_{R}\left(\mathfrak{p}_{i}\right)} \mathfrak{p}_{i}+\sum_{i=s+1}^{t} \frac{n_{i}}{e_{R}\left(\mathfrak{p}_{i}\right)} \mathfrak{p}_{i} \quad \text { and } \quad E_{2}=\sum_{i=s+1}^{t} \frac{n_{i}^{\prime}}{e_{R}\left(\mathfrak{p}_{i}\right)} \mathfrak{p}_{i}+\sum_{i=t+1}^{m} \frac{d_{D}}{e_{R}\left(\mathfrak{p}_{i}\right)} \mathfrak{p}_{i}
$$

where $n_{i}+n_{i}^{\prime}=d_{D}$ for $s+1 \leq i \leq t$. Without loss of generality, we may assume that $n_{s+1}$ is minimal in $\left\{n_{s+1}, \cdots, n_{t}, n_{s+1}^{\prime}, \cdots, n_{t}^{\prime}\right\}$. We put $E=E_{1}+\sum_{j=t+1}^{m} \mathfrak{p}_{j}-n_{s+1} D \in \underline{\mathrm{P}}(R)$. Then $E \ngtr E_{i}$ for $i=1,2$. Since $E=\{E\}$, there exists $E^{\prime} \in \operatorname{Fund}(R) \backslash\left\{D, E_{1}, E_{2}\right\}$ such that $E^{\prime} \leq E$ and $\operatorname{Supp}\left(E^{\prime}\right) \cap\left\{\mathfrak{p}_{1}, \cdots, \mathfrak{p}_{s}\right\} \neq \varnothing$. We note that, for every $E^{\prime \prime} \in \operatorname{Fund}(R) \backslash$ $\left\{D, E_{1}, E_{2}\right\}$, either $\operatorname{Supp}\left(E^{\prime \prime}\right) \subset \operatorname{Supp}\left(E_{1}\right)$ or $\operatorname{Supp}\left(E^{\prime \prime}\right) \subset \operatorname{Supp}\left(E_{2}\right)$. Hence $\operatorname{Supp}\left(E^{\prime}\right) \subset$ $\operatorname{Supp}\left(E_{1}\right)$ and $E^{\prime} \leq \sum_{\mathfrak{p} \in \operatorname{Supp}\left(E_{1}\right)} E(\mathfrak{p})<E_{1}$. This contradicts $E_{1} \in \operatorname{Fund}(R)$.

The proof of Claim 1 is completed. 
We $\operatorname{put}\left\{\mathfrak{p}_{1}, \cdots, \mathfrak{p}_{q}\right\}=\operatorname{Supp}(D) \backslash \bigcup_{i=1}^{p} \operatorname{Supp}\left(E_{i}\right)$.

Claim 2. $\quad w(D) D=\sum_{i=1}^{p} E_{i}+\sum_{j=1}^{q} \mathfrak{p}_{j}$ for some $w(D)>0$.

There exists an integer $w>0$ such that $E:=\sum_{i=1}^{p} E_{i}+\sum_{j=1}^{q} \mathfrak{p}-w D \geq 0$ and $\operatorname{Supp}(E) \subsetneq \Lambda$. If $E \neq 0$, then there exists $E^{\prime} \in \operatorname{Fund}(R) \backslash\{D\}$ such that $E^{\prime} \leq E$. On the other hand, we have $\operatorname{Supp}\left(E^{\prime}\right) \subset \operatorname{Supp}\left(E_{i}\right)$ for some $i$. $\operatorname{Since} \operatorname{Supp}\left(E_{i}\right) \cap \operatorname{Supp}\left(E_{j}\right)=\varnothing$ $(i \neq j), E^{\prime} \leq \sum_{\mathfrak{p} \in \operatorname{Supp}\left(E^{\prime}\right)} E(\mathfrak{p}) \mathfrak{p}<E_{i}$. This is a contradiction. Hence $E=0$ and $w D=$ $\sum_{i=1}^{p} E_{i}+\sum_{j=1}^{q} \mathfrak{p}$.

Combining Claims 1,2 and the induction hypothesis, we have that $\operatorname{Fund}(R)=$ $\{D\} \cup \bigcup_{i=1}^{p} \operatorname{Fund}\left(R^{\left.\left(G_{\text {Supp(E }}\right)\right)}\right)$ is a datum.

(2) We prove that $J_{\Lambda}=\left(Y_{E}^{w(E)}-a_{E} \prod_{E^{\prime}<E} Y_{E^{\prime}} \mid E \in\right.$ Fund $\left.(R)\right)$ by induction on $|\operatorname{Fund}(R)|$. If $\operatorname{Fund}(R)=\varnothing$, then there is nothing to prove. We assume that $\operatorname{Fund}(R) \neq \varnothing$. Let $E \in \operatorname{Fund}(R)$ such that $\operatorname{Supp}(E)$ is maximal in $\{\operatorname{Supp}(D) \mid D \in$ Fund $(R)\}$. Then it suffices to show that locally a complete intersection $R^{\left(G_{\text {Supp(E)) }}\right.}$ satisfies the above condition. Therefore we may assume that $E=\sum_{\mathfrak{p} \in A}\left(1 / e_{R}(\mathfrak{p})\right) \mathfrak{p} \in \operatorname{Fund}(R)$. We put $\left\{E_{1}, \cdots, E_{p}\right\}=\{D \in \operatorname{Fund}(R) \mid D \prec E\}$. Then, by induction hypothesis, $R^{\left(G_{\text {supp }\left(E_{i}\right)}\right)}$ satisfies the assertion (2) $(1 \leq i \leq p)$. Then we have only to show that $J_{\Lambda}=\left(Y_{E}^{w(E)}-\right.$ $\left.a_{E} \prod_{i=1}^{p} Y_{E_{i}}\right)+\sum_{i=1}^{p} J_{\operatorname{Supp}\left(E_{i}\right)}$.

Let $E_{1}^{\prime}, \cdots, E_{t}^{\prime} \in$ Fund $(R) \backslash\{E\}$. Assume that there exists a relation

$$
\underline{\operatorname{div}}_{A}(a)+d E+\sum_{i=1}^{s} d_{i} E_{i}^{\prime}=\underline{\operatorname{div}}_{A}(b)+\sum_{i=s+1}^{t} d_{i} E_{i}^{\prime}
$$

Then, by definition of a datum, $E_{1}^{\prime}, \cdots, E_{t}^{\prime} \in \bigoplus_{\mathfrak{p} \in \Lambda} \mathbf{Z}\left(w(E) / e_{R}(\mathfrak{p})\right) \mathfrak{p}$. Hence $d E \epsilon$ $\bigoplus_{p \in A} Z\left(w(E) / e_{R}(\mathfrak{p})\right) \mathfrak{p}$ and $w(E)$ divides $d$. This implies $J_{\Lambda}=\left(Y_{E}^{w(E)}-a_{E} \prod_{i=1}^{p} Y_{E_{i}}\right)+$ $\sum_{i=1}^{p} J_{\operatorname{Supp}\left(E_{i}\right)}$.

The assertion (3) follows from definition of $G(\operatorname{Fund}(R))$.

\section{Abelian extensions which are complete intersections.}

Let $A$ be a Noetherian normal domain with $K=Q(A)$ and $L$ be a finite Abelian extension of $K$ with $G=\operatorname{Gal}(L / K)$. An integral closure $R$ of $A$ in $L$ is called an Abelian extension of $A$ with a Galois group $G$.

REMARK 4.1. We put $\hat{G}=\operatorname{Hom}(G, U(A))$, where $U(A)$ is the multiplicative group of units of $A$. Assume that $\operatorname{ch}(A)$ does not divide $|G|$, if $\operatorname{ch}(A)$ is positive and $A$ contains a primitive $|G|$-th root of unity. Then $R$ can be regarded as $\hat{G}$-graded ring in the following sense.

For $g \in \hat{G}$, we set $R_{g}=\{a \in R \mid \sigma(a)=g(\sigma) a$ for every $\sigma \in G\}$. Then

(1) $R_{0}=R^{G}=A$,

(2) $R_{g} R_{h} \subset R_{g+h}$ for every $g, h \in \hat{G}$,

(3) $R=\sum_{g \in \hat{G}} R_{g}=\bigoplus_{g \in \hat{G}} R_{g}$.

(See 2 of Itoh [8].) 
As a consequence of (3.11), we have the following.

THEOREM 4.2. Let $A$ be a complete intersection factorial local domain and $G$ be a finite Abelian group of $n=|G|$. Assume that

(i) either $\operatorname{ch}(A)=0$ or $\operatorname{ch}(A)=p>0$ and $(p, n)=1$,

(ii) $A$ contains a primitive $n$-th root of unity.

Let $(R, n)$ be a local ring such that $R \supset A$. Then the following are equivalent.

(1) $R$ is an Abelian extension of $A$ with Galois group $G$ such that $R / \mathfrak{n} \cong A / \mathrm{m}$ and is a complete intersection.

(2) There exists a datum $(\Gamma, w)\left(\right.$ in $\left.\operatorname{Div}(A)_{\mathbf{Q}}\right)$ such that $G \cong G(\Gamma)$ and $R \cong R(\Gamma)$.

Proof. (1) $\Rightarrow$ (2): This follows from (4.1) and (3.11). (2) $\Rightarrow$ (1): Let $E \in \Gamma$ such that $\operatorname{Supp}(E)$ is maximal in $\{\operatorname{Supp}(D) \mid D \in \Gamma\}$ and $\left\{E_{1}, \cdots, E_{s}\right\}=\{D \in \Gamma \mid D<E\}$. By Proposition 1.12 of Tomari-Watanabe [14], if $R\{\Gamma \backslash\{E\})$ is normal domain, then so is

$$
R(\Gamma) \cong R(\Gamma \backslash\{E\})\left[Y_{E}\right] /\left(Y_{E}^{d_{E}}-a_{E} \prod_{i=1}^{s} y_{E_{i}}\right)
$$

Hence $R$ is normal domain. Also, by assumptions (i) and (ii), we have $Q(R)$ is Galois extension of $K$ and $G a l(Q(R) / K) \cong G(\Gamma) \cong G$.

ACKNOWLEDGEMENT. The author would like to express his appreciation to Doctor Kazuhiko Kurano for stimulating discussion. Thanks are also due to Professor Kei-ichi Watanabe and Professor Shiro Goto for several useful suggestions.

\section{References}

[ 1 ] D. F. Anderson, Graded Krull domains, Comm. Algebra 7 (1979), 79-106.

[2] L. Avramov, Flat morphisms of complete intersections, Soviet Math. Dok1. 16 (1975), $1413-1417$.

[3] N. Bourbaki, Algèbre Commutative, Hermann (1965).

[4] M. Demazure, Anneaux gradues normaux, Seminaire Demazure-Giraud-Teissier, Singularites des Surfaces, Ecole Polytechnique (1979), 35-68.

[ 5 ] K. Ето, Ideals associated with free abelian groups, preprint (1994).

[6] S. Goto and K.-i. Watanabe, On graded rings, J. Math. Soc. Japan 30 (1978), 172-213.

[ 7 ] P. Griffith, Normal extensions of regular local rings, J. Algebra 105 (1987), 465-475.

[8] S. Ітон, Cyclic Galois extensions of regular local rings, Hiroshima Math. J. 19 (1989), 309-318.

[9] Y. KAMOI, Noetherian rings graded by an Abelian group, Tokyo J. Math. 18 (1995), 31-48.

[10] H. Nakajima, Affine torus embeddings which are complete intersections, Tôhoku Math. J. 38 (1986), 85-98.

[11] C. NǍstǍSESCU and F. Van OYSTAEYEN, Graded Ring Theory, North-Holland (1982).

[12] P. Roberts, Abelian extensions of regular local rings, Proc. Amer. Math. Soc. 78 (1980), 307-310.

[13] L. Robilano, Introduction to the Theory of Gröbner Basis, Qeen's Papers in Pure and Appl. Math. 80 (1988).

[14] M. Tomari and $\mathrm{K}$.-i. Watanabe, Normal $\mathbf{Z}_{\boldsymbol{r}}$-graded rings and normal cyclic covers, Manuscripta Math. 76 (1992), 325-340. 
[15] K.-i. WATANBE, Invariant subrings which are complete intersections, I, Nagoya Math. J. 77 (1980), 89-98.

[16] K.-i. WATANABE, Some remarks concerning Demazure's construction of normal graded rings, Nagoya Math. J. 83 (1981), 203-211.

Present Address:

Department of Mathematics, Tokyo Metropolitan University, Minami-OHSAWA, HaChioji-SHI, ToKyo, 192-03 JaPAN.

e-mail: kamoi@math.metro-u.ac.jp 